\title{
Perfil e condições de trabalho de agentes comunitários de saúde em município mineiro endêmico para doenças negligenciadas transmitidas por vetores
}

\section{Profile and working conditions of community health agents in endemic municipality for neglected diseases transmitted by vectors}

\begin{abstract}
Como citar este artigo:
REZENDE, CECÍLIA N.; FERREIRA, RAQUEL A.; DOS SANTOS, LORENA

P.; NOGUEIRA, MARIA JOSÉ; Perfil e condições de trabalho de agentes comunitários de saúde em município mineiro endêmico para doenças negligenciadas transmitidas por vetores. Revista Saúde (Sta. Maria). 2020; 46 (2).
\end{abstract}

\section{Autor correspondente:}

Nome: Cecilia Nogueira Rezende

E-mail: cecilia.nogueira@gmail.com Telefone: (31) 983238648

Formação Profissional: Formada em Ciências Sociais pela Universidade Federal de Minas Gerias (UFMG) que fica na cidade de Belo Horizonte, Minas

Gerais, Brasil

Filiação Institucional:Mestranda do Programa de Pós Graduação em Saúde Pública da Universidade Federal de Minas Gerais

Endereço para correspondência: Rua: Aquiri, n 120

Bairro: Aarão Reis

Cidade: Belo Horizonte

Estado: Minas Gerais

CEP: 31814-330

Data de Submissão:

22/05/2020

Data de aceite:

25/11/2020

Conflito de Interesse: Não há conflito de interesse

\section{(c) $)$ BY-NC-ND}

\author{
Cecília Nogueira Rezende, Raquel Aparecida Ferreira, Lorena Portela dos Santos, Maria José \\ Nogueira
}

RESUMO

Objetivo: traçar o perfil dos agentes comunitários de saúde (ACSs) do município mineiro de Sabará, endêmico para várias doenças negligenciadas transmitidas por vetores (DNTVs), e analisar a percepção dos gerentes das UBSs sobre as DNTVs, e também a respeito da inserção e condições de trabalho do agente comunitário de saúde ACS nas equipes multiprofissionais. Métodos: foi aplicado aos ACSs um formulário estruturado, levantando o seu perfil profissional e sociodemográfico. Também foram realizadas entrevistas semiestruturadas com os gerentes das UBSs. Resultados: a maioria dos ACSs eram mulheres jovens com ensino médio completo, há dois anos ou menos na função, e com reduzida participação em cursos de capacitação. Observou-se através da fala dos gerentes um desconhecimento conceitual sobre a definição de doenças negligenciadas. Em relação a inserção dos ACSs, os gerentes reconhecem o papel destes profissionais como elo entre a equipe e a comunidade. Os participantes também identificaram situações que contribuem para a precarização do vínculo profissional dos ACSs na Atenção Primária à Saúde (APS), destacando a baixa remuneração dos profissionais, contratações temporárias e curtas, alta rotatividade, desvio de função e extrapolação das relações profissionais para o âmbito pessoal por parte da população. Considerações finais: através deste estudo foi possível observar que apesar dos desafios de inserção dos ACSs nas equipes multiprofissionais e das precárias condições de trabalho, sua presença é de suma importância no contexto da APS, especialmente ao que tange a prevenção das DNTVs. Sugere-se que os achados deste estudo representem a realidade de outras regiões do Brasil.

PALAVRAS-CHAVE: Doenças Negligenciadas; Atenção Primária à Saúde; Estratégia Saúde da Família; Agentes Comunitários de Saúde.

\section{ABSTRACT}

Objectives: This study aimed to outline the community health workers (CHWs) profile in the municipality of Sabará, Minas Gerais, endemic for several neglected vector-borne diseases (NVBDs), to analyze the perception of UBS managers about NVBDs, and also regarding the insertion and the working conditions of CHWs in multi-professional teams. Methods: A structured form was applied to CHWs, raising their professional and sociodemographic profile. The UBS managers passed through semi-structured interviews. Results: The majority of CHWs were young women with complete high school education, with roughly two years of experience and no participation in training courses for the position held. The manager's speech appointed a misuse of the neglected disease concept. Regarding the insertion of CHWs, managers recognized the role of these professionals as a link between the team and the community. Participants also identified situations that contribute to the precariousness of the professional bond of $\mathrm{CHWs}$ in Primary Health Care (PHC), highlighting the low remuneration of professionals, temporary and short hires, high turnover, job deviation, and extrapolation of professional relationships to the scope staff on the part of the population. Final Considerations: Through this study, it was possible to observe that despite the challenges of inserting $\mathrm{CHWs}$ in multi-professional teams and the precarity of the working conditions, their presence is of paramount importance in the context of PHC, especially concerning the prevention of DNTVs. The findings of this study suggest a representation of the reality in other regions of Brazil.

KEYWORDS: Neglected Diseases; Primary Health Care; Family Health Strategy Community Health Workers. 


\section{INTRODUÇÃO}

As doenças elencadas como negligenciadas pela organização mundial de saúde estão presentes, atualmente, em 149 países, afetando mais de um bilhão de pessoas. As populações que vivem na pobreza, sem saneamento adequado e em contato próximo com vetores infecciosos e animais domésticos e gado são as mais afetadas ${ }^{1}$. Diversas doenças negligenciadas possuem relevância no cenário epidemiológico brasileiro e, consequentemente, para a saúde pública, especialmente, aquelas transmitidas por vetores, uma vez que o Brasil é um país de clima tropical, e portanto, apresenta uma fauna de vetores rica e numerosa ${ }^{2}$. No que se refere ao diagnóstico, tratamento e prevenção dessas doenças, as ações são solucionadas no Sistema Único de Saúde (SUS), sendo que aquelas de caráter preventivo e de controle de vetores se situam no âmbito da Atenção Primária à Saúde (APS) e na vigilância em saúde, respectivamente ${ }^{3}$.

Em países tropicais, como o Brasil, é de grande destaque doenças negligenciadas que apresentam vetores como veiculadores de patógenos. Estas enfermidades possuem uma relação intrínseca com o meio ambiente. Portanto, o conhecimento adequado do território e das famílias pode ser determinante para o planejamento efetivo de ações de prevenção, diagnóstico e tratamento das Doenças Negligenciadas Transmitidas por Vetores (DNTVs), e para colaborar com a vigilância em saúde nas ações de controle. Neste sentido, o Plano de Ação Sobre Entomologia e Controle de Vetores (2018-2023) inclui dentro de linhas de ações importantes o engajamento e mobilização de comunidades e serviços de saúde com atuação local, bem como a melhoria na vigilância dos vetores ${ }^{4}$. Desta forma, ações desenvolvidas pela equipe de saúde da família com participação da comunidade, focando as necessidades locais e buscando resolver os desafios apresentam grande potencial ${ }^{5}$.

Desde o surgimento da profissão de ACS no âmbito do SUS, o grande diferencial foi a seleção de pessoas com perfil adequado para o desempenho das funções e que fossem moradores da localidade de atuação ${ }^{6}$. Baptistini e Figueiredo ${ }^{6}$ destacam que o ACS enquanto morador da localidade em que trabalha facilita a identificação das necessidades de saúde da população, uma vez que ele compartilha do mesmo contexto social e cultural e do mesmo universo linguístico.

O Plano Nacional de Controle da Dengue ${ }^{7}$, lista como uma das atribuições do ACS a atuação junto aos domicílios, passando informações aos moradores sobre a doença, seus sintomas, riscos, forma de transmissão, e também conscientizando sobre o vírus e o vetor da doença. No caso da leishmaniose visceral (LV), o Ministério da Saúde preconiza que os ACSs devem ser capacitados para informar à população acerca dos sintomas, da cadeia e da forma de transmissão desta doença, além dos cuidados coletivos e individuais necessários ao bloqueio da transmissão do parasito, focando principalmente nas condições ambientais que propiciam a proliferação do vetor ${ }^{8}$. Outro exemplo de DNTV em que é primordial o papel do ACS é a leishmaniose tegumentar americana (LTA). Nos guias epidemiológicos do Ministério da Saúde (MS) é preconizado que os ACSs devam observar o aparecimento de lesões sugestivas de LTA nos 
residentes sobre as suas supervisões ${ }^{9}$.

Dentro do sistema de saúde brasileiro os ACS atuam na APS, esse nível de atenção tem presença estratégica nos territórios, sendo considerado a porta de entrada preferencial de todos os usuários ao sistema de saúde ${ }^{3}$. É esperado que a atenção básica seja resolutiva para 85\% dos problemas de saúde de uma população, além de ser responsável por ordenar os fluxos de pacientes e informações dentro da Rede de Atenção à Saúde (RAS) ${ }^{10}$.

Neste contexto de trabalho em rede surgiu o Programa Saúde da Família (PSF), que em razão da sua amplitude passou a ser chamado Estratégia Saúde da Família $(E S F)^{10}$. Um dos objetivos da ESF é reorganizar o sistema de saúde com base na APS, melhorando o estado da saúde da população mediante a construção de um modelo assistencial de atenção orientado para os determinantes do processo saúde-doença, considerando contextos culturais e sociais ${ }^{11}$.

As equipes de ESF, para que alcancem a complexidade dos objetivos propostos, podem ser formadas por médicos generalistas, enfermeiros, técnicos ou auxiliares de enfermagem e ACS. Outro profissional, que não compõe a equipe da ESF, mas que tem um papel importante para a gestão e funcionamento adequada das unidades básicas de saúde (UBS) e unidades de saúde da família (USF) é o gerente. As principais atribuições desse profissional são 0 planejamento em saúde, a gestão e organização do processo de trabalho, bem como a coordenação das ações no território e integração da Unidade de Saúde da Família (USF) como os outros serviços da RAS ${ }^{3}$. É importante destacar, também, que a função de gerência normalmente é realizada por um integrante da equipe que acaba acumulando duas funções ${ }^{12}$.

Diante do exposto acima, e do evidente papel do ACS no contexto de prevenção das DNTVs, o presente estudo teve como objetivo traçar o perfil e conhecer as condições de trabalho dos ACSs do município mineiro de Sabará, endêmico para várias DNTVS ${ }^{13}$. Além disso, analisar a percepção dos gerentes das UBSs sobre as DNTVs e a inserção dos ACSs nas equipes multiprofissionais. Na literatura científica não há estudos relacionados ao entendimento do conceito de doenças negligenciadas por profissionais da ESF. Desta maneira, o presente trabalho contribui para essa compreensão, sendo de grande relevância, pois as práticas de cuidado dos pacientes e as ações de prevenção e controle dessas doenças serão medidas por essas construções conceituais. Por outro lado, o artigo também contribui para a construção do conhecimento sobre a profissão de ACS em âmbito local em diálogo com outros estudos da realidade brasileira.

\section{MÉTODO}

Trata-se de uma pesquisa quanti-qualitativa, desenvolvida em Sabará-MG, município localizado na região metropolitana de Belo Horizonte, Minas Gerais.

Participaram do projeto, os integrantes das UBS que possuíam, pelo menos, uma equipe de ESF completa e em 
funcionamento durante o período de junho a julho de 2014. Desse modo, ao final foram elencadas, nove UBS das 16

presentes no município. Para traçar o perfil dos ACSs foi elaborado um formulário estruturado com as seguintes variáveis: sexo, idade, escolaridade, tempo no cargo de ACS e se o mesmo já realizou qualificações ou capacitações para ocupar o cargo de ACS. Os dados foram processados e digitalizados no programa Microsoft Excel $(2010)$, e posteriormente foram calculadas as frequências absolutas e relativas dos dados.

Além disto, foram entrevistados os gerentes dessas nove UBS, uma vez que esses profissionais são os responsáveis por acompanhar as ações dos integrantes da ESF na APS. O instrumento de coleta de dados utilizado foi um roteiro semiestruturado de entrevista, contendo perguntadas relacionadas aos eixos: perfil do participante, trajetória profissional, inserção e condições de trabalho dos ACSs.

As entrevistas foram agendadas e ocorreram nas próprias UBS dos respondentes, no período de março a abril de 2015. Foram gravadas com consentimento dos entrevistados, transcritas e analisadas sob a perspectiva de análise de conteúdo de Bardin ${ }^{14}$ em três fases cronológicas. Identificaram-se unidades de análise em cada entrevista e entre as entrevistas e nomearam-se as seguintes categorias: a) ACS como categoria profissional no contexto da APS e sua inserção na ESF, b) DNTV e o papel do ACS no âmbito da prevenção e promoção da Saúde.

Ressalta-se que a presente proposta foi submetida à apreciação pelo comitê de ética em pesquisa do Centro de Pesquisas Renê Rachou sob o número de CAAE: 27298014.6.0000.5091 e a mesma foi aprovada em 27 de fevereiro de 2014.

\section{RESULTADO E DISCUSSÃO}

\section{Perfil do Agente Comunitário de Saúde}

À época da pesquisa, o município de Sabará contava com 63 ACSs pertencentes as nove UBSs com ESF existentes no município, desse total, 52 profissionais preencheram o formulário e o perfil deles pode ser observado na tabela 1. 
Tabela 1. Perfil dos Agentes Comunitários de Saúde de Sabará/MG, 2014

\begin{tabular}{|c|c|c|c|}
\hline PARÂMETRO & CATEGORIA & FREQUÊNCIA & PORCENTAGEM (\%) \\
\hline \multirow[t]{2}{*}{ Sexo } & Feminino & 48 & 92,0 \\
\hline & Masculino & 4 & 8,0 \\
\hline \multirow[t]{2}{*}{ TOTAL } & & 52 & 100,0 \\
\hline & $21-25$ & 5 & 9,6 \\
\hline \multirow[t]{7}{*}{ Idade em anos } & $26-30$ & 12 & 23,1 \\
\hline & $31-35$ & 10 & 19,2 \\
\hline & $36-40$ & 12 & 23,1 \\
\hline & $41-45$ & 2 & 3,9 \\
\hline & $46-50$ & 5 & 9,6 \\
\hline & $50 \geq$ & 5 & 9,6 \\
\hline & Em branco & 1 & 1,9 \\
\hline \multicolumn{2}{|l|}{ TOTAL } & 52 & 100,0 \\
\hline \multirow{2}{*}{$\begin{array}{l}\text { Ensino médio } \\
\text { completo }\end{array}$} & $\operatorname{Sim}$ & 46 & 88,0 \\
\hline & Não & 6 & 12,0 \\
\hline \multicolumn{2}{|l|}{ TOTAL } & 52 & 100,0 \\
\hline \multirow{3}{*}{$\begin{array}{l}\text { Curso de } \\
\text { qualificação }\end{array}$} & Sim & 24 & 46,2 \\
\hline & Não & 23 & 44,2 \\
\hline & Em branco & 5 & 9,6 \\
\hline \multirow[t]{2}{*}{ TOTAL } & & 52 & 100,0 \\
\hline & $\leq 1$ & 14 & 27 \\
\hline \multirow{3}{*}{$\begin{array}{c}\text { Tempo de } \\
\text { serviço (anos) }\end{array}$} & $1-2$ & 26 & 50 \\
\hline & $3-5$ & 3 & 6 \\
\hline & $6 \geq$ & 9 & 17 \\
\hline TOTAL & & 52 & 100 \\
\hline
\end{tabular}

No município estudado, $92 \%$ dos ACSs que preencheram o formulário (tabela 1) eram mulheres, corroborando com dados da literatura ${ }^{15}$. Georges e Santos ${ }^{15}$ observaram um papel social da função de ACS dentro das comunidades, muitas mulheres encontraram uma oportunidade de inserção profissional, que significava além de uma renda para família, um caminho para emancipação feminina.

Quanto a faixa etária, predominou no estudo profissionais jovens adultos, uma vez que dentre aqueles ACSs que responderam à questão, $67 \%$ dos ACSs possuíam idade entre 26 a 40 anos. Mota e David ${ }^{16}$ encontraram o mesmo predomínio desta faixa etária em seu estudo, os autores destacam que a ampliação da presença de ACS em áreas 
urbanas são os fatores que colaboraram para que o perfil etário passasse a atrair os mais jovens nos processos seletivos.

Quanto ao grau de escolaridade, 88\% dos participantes afirmaram possuir o ensino médio completo (tabela 1), 0 que está de acordo com o preconizado pelo MS, que passou a exigir, em 2002, como requisito para exercer essa função, a conclusão do ensino fundamental ${ }^{17}$.

No que diz respeito à formação para exercício da profissão de ACS, dentre aqueles que responderam a essa questão, 49\% afirmaram não ter passado por qualificação ou capacitação, apontando uma discrepância com 0 preconizado pelo MS, que estabelece como condição para realização do trabalho de ACS a participação em um curso profissionalizante próprio para o exercício das funções de $\mathrm{ACS}^{3}$. Dos $51 \%$ que responderam a questão e afirmaram possuir alguma qualificação, a maioria não soube informar qual foi o tipo de qualificação. De fato, Morosini, Corbo e Guimarães ${ }^{18}$ pontuam que o ACS acumula em sua trajetória uma história marcada pela precarização de vínculos e pelo aligeiramento de sua formação. Dentre as profissões que compõem a equipe multiprofissional a de ACS é a única que não exige uma formação pregressa dos candidatos, e é após o processo de seleção que o profissional recebe uma capacitação para desempenho de suas funções ${ }^{19}$. Entretanto, em Sabará, à época do estudo, nem mesmo esta capacitação pós ingresso no serviço parece ter sido ofertada aos profissionais. Ainda, segundo Gomes et al. ${ }^{20}$ o processo de formação do ACS exige um esforço de capacitação permanente, que tenha como base os princípios do SUS e a construção compartilhada do conhecimento, buscando compreender as experiências dos profissionais dentro dos territórios, para desta forma orientar o trabalho.

O município possui uma variação considerável no tempo em que os ACS estão no cargo, variando entre 3 meses a 16 anos (tabela 1). Contudo, é importante destacar que 77\% deles ocupam a função há 2 anos ou menos (tabela 1). Dessa forma, a maioria deles ainda não passou pelo vencimento do processo seletivo, que é de 2 anos.

\section{Doenças negligenciadas transmitidas por vetores e o papel do ACS no âmbito da prevenção e promoção}

\section{da Saúde}

De acordo com a ANVISA ${ }^{21}$ as doenças negligenciadas são àquelas que não apresentam atrativos econômicos para o desenvolvimento de fármacos, quer seja por sua baixa prevalência na população como um todo, ou por atingirem populações localizadas em regiões de baixo nível de desenvolvimento. Notou-se, pela fala de alguns entrevistados que há uma compreensão acerca do sentido do termo negligência, no entanto, eles não atribuíram a negligência aos aspectos econômicos, como falta de investimento e ausência de políticas governamentais:

"Negligenciadas (pausa) é que não é percebido, não é abordado da forma como deveria, pra mim é minha opinião" (Gerente 4). 
O que prevaleceu foi uma associação entre a causa das doenças negligenciadas e o descuido ou desatenção dos próprios indivíduos acometidos pelas doenças:

"[...] E sem contar também que essas pessoas, a responsabilidade primeiro é delas né!? De tomar à medicação, de fazer o uso correto, de tudo [...]" (Gerente 9).

"Doença negligenciadas, eu acho que são as doenças que as pessoas não dão muito valor... acho que é mais pro lado de não dá valor mesmo, não cuida direito, não leva o cão pra vacinar [...]" (Gerente 5).

Quanto a culpabilização da vítima, o lócus de responsabilidade e a unidade de análise é o indivíduo, que é visto como o último responsável (senão o único) por seu estado de saúde. Schmidt ${ }^{22}$ chama atenção para as consequências desse tipo de atitude, à medida que o próprio sujeito é unicamente encarregado pela situação social, sanitária e de saúde em que se encontra, os poderes públicos acabam sendo desvinculados das reais responsabilidades que possuem, sobretudo em relação aos serviços de prevenção. Freitas e Mandú ${ }^{23}$ destacam que a prevenção adequada deve ocorrer de forma compartilhada entre o poder público e a população, usando uma linguagem simples e de caráter educativo.

O Ministério da Saúde ao estabelecer as atribuições dos ACSs coloca como aspecto estruturante de suas funções, o papel de educador, de agente de mudanças nas comunidades ${ }^{11}$. Ferraz e Aerts ${ }^{24}$ estudaram o cotidiano de trabalho de ACSs e classificaram a visita domiciliar, a educação em saúde e o acompanhamento de grupos de risco como as atividades mais desempenhadas por estes profissionais. Os entrevistados destacaram a importância de os ACSs possuírem conhecimentos sobre as doenças negligenciadas, conforme destacado abaixo, reconhecendo assim, 0 potencial que estes profissionais possuem na transmissão e repasse de informações:

"Acho que eles têm que ter um maior conhecimento sobre cada doença específica [...] é importante o trabalho do agente de saúde, nas doenças negligenciadas eu acho mais importante ainda... eles estão nas casas do paciente, então se eles souberem orientar bem as pacientes sobre os cuidados diários, ajuda bastante na prevenção" (Gerente 5).

"Porque uma vez que o ACS faz uma visita, vê aquela pessoa, ele já vai saber os sintomas de uma dengue né... e já vão acionar a unidade de saúde... eles que estão vendo o que tá acontecendo" (Gerente 6). 
Conforme mencionado faz parte das atribuições do ACS realizar visita domiciliar. Essa função confere ao profissional maior proximidade com a população e implica na construção de vínculos, que quando consolidados garantem laços de confiança e corresponsabilidade no trabalho dos profissionais junto aos usuários. Neste sentido, os ACSs desempenham um papel de mediação, são eles que transmitem as necessidades de saúde da população para a equipe de saúde e também são estes profissionais que levam informações aos usuários ${ }^{12}$. Portanto, a criação de laços efetivos entre ACS e população pode ser um facilitador para ações de prevenção e promoção de saúde. Na perspectiva dos gerentes existe no município um elo forte entre os ACSs e a comunidade:

"[...] o vinculo aqui é enorme. Essa construção de vínculos deles é bem forte, já é consolidada né" (Gerente 6).

"Ah, o vínculo com agente é excelente assim. São bem inteirados assim, conversam bastante. Todo mundo se conhece" (Gerente 4).

No âmbito das doenças negligenciadas transmitidas por vetores a atuação do ACS junto à comunidade enquanto agente educador representa uma potencialidade na APS. No entanto, a PNAB de 2017 abre precedentes para redução no número de ACS nas equipes, não estabelecendo mais um quantitativo obrigatório ${ }^{3}$. A política estabelece, também, novas competências do ACS, contribuindo para descaracterizar a função, tornando-a mais técnica do que comunitária ${ }^{25}$.

\section{ACS como categoria profissional no contexto da APS}

Um dos principais pré-requisitos estabelecido pelo MS para pleitear o cargo de ACS é a obrigatoriedade de morar na comunidade onde pretende atuar ${ }^{20}$. Cardoso e Nascimento ${ }^{26}$ destacam que esse contato permanente que o ACS tem com as famílias, este vínculo, pode facilitar o trabalho de vigilância e promoção da saúde que é realizado por toda a equipe multiprofissional na ESF. Tal fato é elucidado nas falas dos entrevistados:

"[...] porque eles são membros da população, então eles têm uma, eh, uma amizade, uma afinidade muito grande [...]" (Gerente 7).

"E o agente de saúde traz pra gente a demanda, eles têm adquirido o vínculo com os pacientes [...]" (Gerente 5). 
Em contrapartida, Jardim e Lancman²7, apontam que a dupla inserção na comunidade leva o ACS a ter duplo papel: 0 de ser simultaneamente agente e sujeito. Tal relação cria uma porosidade entre o trabalhar e 0 viver na comunidade, ou seja, o agente não tem o distanciamento entre as esferas da vida profissional e pessoal. Essa situação foi identificada na fala de alguns gerentes.

"[...]Agora eu vejo também, que precisa muito ser trabalhado isso... hoje já vieram queixar, fizeram até por escrito a reclamação de um amigo, vizinho, compadre que tá ali e ter 0 atendimento priorizado por isso, pelo vínculo do agente..." (Gerente 7).

"São da comunidade e facilita em alguns casos, mas em outros, tem alguns pacientes que não aceita que seja tal agente de saúde que vai na casa dele, ai a gente vai e troca o agente de saúde... por ser da comunidade, tem rinchas pessoais [...]" (Gerente 5).

As situações descritas abrem espaço para uma reflexão sobre os impactos que a profissão de ACS exerce nas relações pessoais e afetivas dos sujeitos nos espaços. Georges e Santos ${ }^{15}$, em estudo sobre a perspectiva de ACS, identificaram que o fato de trabalhar e morar no mesmo local implica em exercer as funções próprias da profissão em tempo integral. Nesse estudo, as ACS relataram que eram procuradas em suas próprias residências nos horários de folga, e que se sentiam obrigadas a prestar assistência, pois tinham como experiência que a negação poderia resultar em rejeição ${ }^{15}$.

Quanto ao vínculo profissional dosACSs, a maioria dos gerentes indicaram situações de fragilidade e precarização. A legislação vigente, na época das entrevistas, previa que a contratação de ACS deveria ser feita por meio de processo seletivo simplificado, com a validade máxima de dois anos, após esse período o profissional deveria ser submetido a nova avaliação. Na visão de Monteiro e Previtali ${ }^{28}$, essas situações tornam o trabalhador vulnerável, sem poder de negociações e escolhas, o que pode ser um causador de sofrimentos, angústias e incertezas.

"[...] inclusive os que estão né, vão completar dois anos agora no final do ano já estão preocupados né, como que vai acontecer, e até mesmo na equipe gera, porque virão novos até pegarem o serviço, então, assim... isso gera uma ansiedade neles [...]" (Gerente 8). 
"[...] fica dois anos, sai, entra outro, até criar o vínculo que eles já adquiriram com a população... o problema é com criação de vínculo, esse fato de é uma profissionaliza/ profissão até muito, pra mim, uma das mais importantes pra PSF, e o fato de ter essa rotatividade, eu acho pro PSF é muito ruim..." (Gerente 7).

Conforme expresso nas falas dos gerentes, essas fragilidades podem implicar na não continuidade do profissional no cargo de ACS e, portanto, levam à maior rotatividade destes profissionais na equipe, o que gera a necessidade de nova criação de vínculo e descontinuidade da atenção. Outros desafios encontrados foram as situações adversas de trabalho e a questão salarial abordadas por alguns gerentes como um problema. Eles destacaram que o salário do ACS é baixo e muitos precisam desenvolver outras funções para complementar a sua renda, conforme destacado nas falas abaixo:

"[...] o salário deles é muito baixo, então muitos deles, eles trabalham com outra coisa, tem uma que é decoradora, tem outra que trabalha com, eh, cerimonial e eventos, então todos eles tem um bico até assim de dar uma faxina aqui ou ali” (Gerente 2).

"Olha eu acho, que esse cargo, esse emprego, que assim é mais um serviço temporário mesmo. Que eu acho que ninguém quer ser agente comunitário a vida toda. Porque é um serviço também pesado né. Igual aqui, aqui é uma área de risco tem muitas ruas aqui que não tem calçamento, o acesso é difícil” (Gerente 3).

Outra perspectiva sobre a produção de mecanismos de desvalorização do trabalho do ACS é trazida por Bornstein $^{29}$, que, identifica práticas de desvio de função deste trabalhador, sobretudo no sentido de responsabilizá-lo pela execução de tarefas burocráticas. Para a autora, a recorrência deste fenômeno deve nos fazer refletir sobre a desvalorização do trabalho do ACS como mediador no trabalho educativo. O desvio de função denota a compreensão do trabalho do ACS como inespecífico e de baixa complexidade, podendo ser redirecionado, segundo as necessidades dos serviços, para atividades que supostamente exigem pouca capacitação.

"São cinco. São cinco sim né!? Aí tem uma em desvio de função e tem uma em afastamento médico" (Gerente 3). 
"Nós temos 5, 6ACS, uma em desvio de função trabalhando na farmácia por causa de um funcionário do administrativo" (Gerente 4).

Imbrizi et al. ${ }^{19}$ afirmam que a atribuição de serviços burocráticos aos ACS, sobrecarregam estes profissionais e prejudicam a qualidade da atenção prestada ao usuário. Prejudicando as funções dos ACSs e seu compromisso com as ações nos domicílios e na comunidade, que apresentam foco na prevenção de doenças e promoção de saúde e que devem ser desenvolvidas por meio de uma lógica educativa, envolvendo a população.

\section{CONSIDERAÇÕES FINAIS}

Através do presente estudo foi possível observar que o perfil do ACS ainda é algo em construção, sendo que a desvalorização social desse trabalhador dificulta à adequação as leis que regem a categoria, explicando, por exemplo, a alta porcentagem de profissionais que não passaram por qualificações ou capacitações no município estudado. Logo, embora seja associado ao ACS um lugar de grande importância no SUS e na ESF, a literatura aponta alguns desafios para consolidação e valorização desse profissional enquanto integrante e integrado a equipe multiprofissional.

Por outro lado, acredita-se que o reconhecimento e a valorização desse profissional veem sendo feita de forma gradativa, com avanços na legislação que apontam para a consolidação da profissão. Alguns desafios, como o processo de formação do ACS, ainda devem ser colocados em pauta e a discussão deveria avançar no sentido de valorizar o seu papel de educador e sua atuação como fortalecedor do vínculo entre a ESF e a comunidade.

A atuação do ACS como educador e facilitador de vínculos pode contribuir para a prevenção e controle do quadro de doenças negligenciadas transmitidas por vetores. Em contextos de emergências públicas, como no caso do Zika vírus e recentemente da pandemia do coronavírus, o papel do ACS junto a população ganha uma importância especial em nível local, pois, mesmo com problemas estruturais graves as ações dos sujeitos aliadas as informações de qualidade podem desempenhar uma rede eficiente de combate aos vetores da doença. Em síntese, a presença do ACS nas equipes de ESF é de suma importância no contexto da APS, especialmente, no auxílio da efetivação de ações territoriais com a população, como acontece no combate as doenças negligenciadas transmitidas por vetores.

Considera-se que as principais limitações desse estudo são inerentes a abordagem qualitativa, que apesar de possibilitar um aprofundamento na temática, dificulta a compreensão do fenômeno em uma perspectiva generalizante. Para pesquisas futuras, em função das recentes alterações da PNAB de 2017, sugere-se verificar os efeitos da política no perfil dos ACSs e na capacidade de atuação comunitária em casos de doenças negligenciadas transmitidas por vetores. 


\section{REFERÊNCIAS}

1. World Health Organization. Neglected tropical diseases. Geneva; 2019. Disponível em: https://www.who.int/ neglected_diseases/diseases/en/. Acesso em 11/11/2020.

2. World Health Organization. The global network for neglected tropical diseases control (GNNTDC). Geneva; 2010.

3. Brasil. Ministério da Saúde. Portaria $n^{0} 2.436$, de 21 de setembro de 2017. Aprova a política nacional de atenção básica, estabelecendo a revisão de diretrizes para a organização da atenção básica, no âmbito do sistema único de saúde (SUS). Diário Oficial da União. 22 Set 2017.

4. Organização Pan-americana da Saúde. Plano de ação sobre entomologia e controle de vetores 2018-2023. Washington, D.C., EUA; 2018.

5. Mendes R, Donato AF. Território: espaço social de construção de identidades e de políticas. Sanare. 2003 fev-mar; IV(1):39-42

6. Baptistini RA, Figueiredo TAM. Agente comunitário de saúde: desafios do trabalho na zona rural. Ambiente \& Sociedade. $2014 ; 17(2): 53-70$.

7. Brasil. Ministério da Saúde. Programa Nacional de Controle da Dengue. Brasília, Funasa, 2002.

8. Secretaria de Estado Saúde de Santa Catarina. Sistema Único de Saúde Superintendência de Vigilância em Saúde. Diretoria de Vigilância Epidemiológica. Gerencia de Vigilância de Zoonoses e Entomologia. Guia de orientação para a vigilância da leishmaniose visceral canina (LVC). 2018.

9. Brasil. Ministério da Saúde. Secretaria de Vigilância em Saúde. Coordenação-Geral de Desenvolvimento da Epidemiologia em Serviços. Guia de Vigilância em Saúde. 3ª ed. Brasília: Ministério da Saúde, 2019.

10. Pinto LF, Giovanella L. Do Programa à Estratégia Saúde da Família: expansão do acesso e redução das internações por condições sensíveis à atenção básica (ICSAB). Ciênc. saúde coletiva. 2018 mar; 23(6):1903-13. 
11. Fertonani HP, Pires DEP, Biff D, Scherer MDA. Modelo assistencial em saúde: conceitos e desafios para a atenção básica brasileira. Ciênc. saúde coletiva. 2015 out; 20(6):1869-78.

12. Lotta GS. Implementação de políticas públicas: o impacto dos fatores relacionais e organizacionais sobre a atuação dos burocratas de nível de rua no Programa Saúde da Família [tese]. São Paulo: Universidade de São Paulo; 2010.

13. Santos, PL., Nogueira, MJ., Rezende, CN., Ferreira, RA. Doenças negligenciadas no município de Sabará: casos, portadores e percepções. Arq. Cienc. Saúde UNIPAR. 2017 set-dez; 21(3):155-162.

14. Bardin L. Análise de Conteúdo. Lisboa: Edições. 2004; (70):89-216.

15. Georges I, Santos YG. As 'novas' políticas sociais brasileiras na saúde e na assistência: produção local do serviço e relações de gênero. Belo Horizonte: Fino Traço, 2016. 355 p.

16. Mota RRA, David HMSL. A crescente escolarização do agente comunitário de saúde: uma indução do processo de trabalho? Trab. educ. saúde. 2010; 8(2): 229-248.

17. Brasil. Ministério da Saúde. Lei no 10.507 de 10 de julho de 2002. Cria a profissão de Agente Comunitário de Saúde e dá outras providências. Diário Oficial da União. 10 Jul 2002.

18. Morosini MV, Corbo ADA, Guimarães CC. O Agente Comunitário de Saúde no âmbito das políticas voltadas para a atenção básica: concepções do trabalho e da formação profissional. Trab. Educ. Saúde. 2007; 5(2):261-280.

19. Imbrizi JM, Aguiar FBT, Fajardo A, Hirata JHB; Kawagoel K; Miyaura AK. Condições de trabalho na Estratégia de Saúde da Família: relato de experiência de extensão universitária com agentes comunitários de saúde. Cad. psicol. soc. trab. 2012 jun; 15(1):153-169

20. Gomes KO, Cotta RMM, Mitre SM, Batista RS, Cherherchiglia ML. O Agente Comunitário de Saúde e a consolidação do Sistema Único de Saúde: reflexões contemporâneas. Physis. 2010; 209(4):1143-1164. 
21. Brasil. Ministério da Saúde. Agência Nacional de Vigilância Sanitária (ANVISA). Resolução RDC $n^{0} 28$, de 4 de abril de 2007. Dispõe sobre a priorização da análise técnica de petições. Diário Oficial [da] República Federativa do Brasil. 2008 mar. 52.

22. Schmidt RAC. A construção do conhecimento do indivíduo no processo de sensibilização-conscientização -ação sobre a hantavirose e a oportunidade para o controle e a prevenção de zoonoses emergentes: a experiência da hantavirose em Santa Catarina/Brasil. Saude soc. 2007 set-dez; 16(3):111-124.

23. Freitas MLA, Mandú ENT. Promoção da saúde na Estratégia Saúde da Família: análise de políticas de saúde brasileiras. Acta paul. enferm. 2010 mar-abr; 23(2):200-5.

24. Ferraz L, Aerts DRGC. O cotidiano de trabalho do agente comunitário de saúde no PSF em Porto Alegre. Ciênc. saúde coletiva [online]. 2005; 10(2):347-355.

25. Melo EA, Mendonça MHM, Oliveira JR, Andrade GCL. Mudanças na Política Nacional de Atenção Básica: entre retrocessos e desafios. Saúde Debate. 2018; 42(Esp. 1):38-51.

26. Cardoso AS, Nascimento, MC. Comunicação no Programa Saúde da Família: o agente de saúde como elo integrador entre a equipe e a comunidade. Ciênc. saúde coletiva. 2010; 15(Supl. 1):1509-1520.

27. Jardim TA, Lancman S. Aspectos subjetivos do morar e trabalhar na mesma comunidade: a realidade vivenciada pelo agente comunitário de saúde. Interface. 2009; 13(28):123-135.

28. Monteiro MAGS, Previtali FS. A política de formação profissional dos agentes comunitários de saúde: limites e possibilidades de construção de sujeitos críticos. Revista Labor. 2011; 1(5):93-115.

29. Bornstein VJ. O agente comunitário de saúde na mediação de saberes. 2007 [Tese Doutorado]. Rio de Janeiro: Escola Nacional de Saúde Pública Sergio Arouca (Ensp)/Fiocruz. 